\title{
Effect of surface roughness on adhesion of graphene membranes
}

\author{
Wei Gao and Rui Huang* \\ Department of Aerospace Engineering and Engineering Mechanics, University of Texas, Austin, \\ $T X 78712$, USA
}

\begin{abstract}
A theoretical model is presented to predict how the surface roughness may affect the adhesion between graphene membranes and their substrates. The bending modulus, which increases drastically from monolayer to multilayered graphene, plays an important role in the transition from conformal to non-conformal morphology of the graphene membranes on a corrugated surface. As such, the measurable adhesion energy drops considerably from monolayer to bilayer graphene. Moreover, the results suggest that tunable adhesion of graphene can be achieved by controlling the surface roughness of the substrate.
\end{abstract}

Keywords: graphene, adhesion, surface roughness 
A recent experiment by Koenig et al. ${ }^{1}$ reported ultra-strong adhesion between graphene membranes and a silicon oxide substrate. In particular, they found that the adhesion energy for monolayer graphene is considerably higher than those for membranes with 2-5 graphene layers. In general, it is essential to understand and characterize interfacial adhesion of graphene membranes on various substrates in order to integrate graphene for practical device applications. Previously, the interaction energy between graphene and silicon oxide $\left(\mathrm{SiO}_{2}\right)$ was estimated to be $0.096 \mathrm{~J} / \mathrm{m}^{2}$ based on the interlayer van der Waals interaction in graphite. ${ }^{2}$ Similar but slightly smaller values were obtained from first-principles calculations for the binding energy of graphene on both O-terminated and hydroxylated $\mathrm{SiO}_{2}$ surfaces. ${ }^{3}$ On the other hand, an experimental measurement by Zong et $a l .{ }^{4}$ reported an average value of $0.151 \mathrm{~J} \mathrm{~m}^{-2}$ for the adhesion energy of multilayered graphene membranes (roughly 5 layers) on silicon, in which they used $0.5 \mathrm{TPa}$ as the Young's modulus of graphene to calculate the adhesion energy based on the measured blister radius and height. However, the Young's modulus of monolayer graphene was measured to be $1.0 \mathrm{TPa},{ }^{5}$ similar to the Young's modulus for the base plane of bulk graphite. ${ }^{6}$ The adhesion energy measured by Koenig et al. ${ }^{1}$ is even higher, $0.31 \mathrm{~J} / \mathrm{m}^{2}$ for multilayered graphene (2 to 5 layers) and $0.45 \mathrm{~J} / \mathrm{m}^{2}$ for monolayer graphene, all on $\mathrm{SiO}_{2}$ surfaces. These values suggest that the interfacial adhesion between graphene and $\mathrm{SiO}_{2}$ is relatively weak compared to covalent bonds. ${ }^{7,8}$ In the present study, a theoretical model is presented by considering the van der Waals interaction between graphene and its substrate. ${ }^{9-11}$ It predicts that the measured adhesion energy depends sensitively on the morphology, which in turn depends on both the substrate surface roughness and the bending modulus of graphene. We find that the difference in the bending moduli between monolayer and multilayered graphene leads to the difference in their adhesion energies. 
On a theoretically flat substrate surface, a graphene membrane remains flat, and the interfacial potential energy (per unit area) due to van der Waals interaction takes the form: ${ }^{11}$

$$
U_{v d W}(h)=-\Gamma_{0}\left[\frac{3}{2}\left(\frac{h_{0}}{h}\right)^{3}-\frac{1}{2}\left(\frac{h_{0}}{h}\right)^{9}\right],
$$

where $h$ is the separation distance between the membrane and the surface, $h_{0}$ is the equilibrium separation, and $\Gamma_{0}$ is the intrinsic adhesion energy per unit area. Here we have assumed that the interfacial van der Waals interaction is predominantly between the substrate and the first graphene layer. The interactions with the other layers, being further apart and shielded by the first layer, are much weaker and thus ignored. On the other hand, the interactions between adjacent graphene layers are assumed to be sufficiently strong to hold the graphene layers together as a continuous membrane. Therefore, on a perfectly flat substrate surface, the interfacial potential energy between a multilayered graphene membrane and the substrate is identical to that for a monolayer graphene, with the same intrinsic adhesion energy $\Gamma_{0}$. However, in experiments the substrate surface is typically corrugated, ${ }^{1,2}$ on which the measurable adhesion energy of graphene depends on the morphology ${ }^{11}$ and becomes different for the monolayer and multilayered membranes.

Consider a periodically corrugated substrate surface (Fig. 1). The van der Waals interaction tends to bring the graphene membrane conformal to the surface. However, corrugation of the membrane increases the elastic strain energy due to bending. The competition between the van der Waals interaction and the bending energy determines the equilibrium morphology of the membrane. Assume a sinusoidal morphology for both the surface and the membrane. The surface roughness is parameterized by the corrugation wavelength $\lambda$ and the 
amplitude $\delta_{s}$. The separation between the membrane and the surface is: $h(x)=h_{m}+\left(\delta_{g}-\delta_{s}\right) \sin (2 \pi x / \lambda)$. For given values of $\lambda$ and $\delta_{s}$, the corrugation amplitude of the membrane $\left(\delta_{g}\right)$ and the mean separation $\left(h_{m}\right)$ are determined by minimizing the total free energy of the system. When $\delta_{g}=\delta_{s}$, the graphene membrane is fully conformal to the surface. When $\delta_{g}=$ 0 , the graphene is flat and non-conformal. As the measurable adhesion energy, the work of adhesion is defined as the energy difference between the equilibrium state and a reference state when the membrane is far away from the surface.

Following the approach by Aitken and Huang, ${ }^{11}$ the van der Waals interaction energy is obtained to the leading order of the corrugation amplitudes as

$$
\tilde{U}_{v d W}\left(h_{m}, \delta_{g}\right) \approx U_{v d W}\left(h_{m}\right)+U_{1}\left(h_{m}\right)\left[\left(\frac{\delta_{g}}{h_{0}}\right)^{2}+\left(\frac{\delta_{s}}{h_{0}}\right)^{2}\right]+U_{2}\left(h_{m}\right) \frac{\delta_{g} \delta_{s}}{h_{0}^{2}}
$$

where

$$
\begin{aligned}
& U_{1}(h)=\frac{9 \Gamma_{0}}{2}\left[-\left(\frac{h_{0}}{h}\right)^{5}+\frac{5}{2}\left(\frac{h_{0}}{h}\right)^{11}\right], \\
& U_{2}(h)=9 \pi^{3} \Gamma_{0}\left[\frac{h_{0}^{5}}{\lambda^{3} h^{2}} K_{3}\left(\frac{2 \pi h}{\lambda}\right)-\frac{\pi^{3} h_{0}^{11}}{24 h^{5} \lambda^{6}} K_{6}\left(\frac{2 \pi h}{\lambda}\right)\right],
\end{aligned}
$$

and $K_{n}(z)$ is the modified Bessel function of the second kind.

The elastic strain energy per unit area of the membrane due to bending is

$$
\tilde{U}_{g}\left(\delta_{g}\right) \approx \frac{D_{N}}{4}\left(\frac{2 \pi}{\lambda}\right)^{4} \delta_{g}^{2}
$$

where $D_{N}$ is the bending modulus of the $N$-layered graphene membrane.

The total free energy of the system is then, $\tilde{U}_{\text {total }}\left(h_{m}, \delta_{g}\right)=\tilde{U}_{v d W}\left(h_{m}, \delta_{g}\right)+\tilde{U}_{g}\left(\delta_{g}\right)$. By setting $\partial \tilde{U}_{\text {total }} / \partial \delta_{g}=0$ and $\partial \tilde{U}_{\text {total }} / \partial h_{m}=0, h_{m}$ and $\delta_{g}$ are obtained simultaneously for the 
equilibrium state. ${ }^{11}$ The normalized results, $h_{m} / h_{0}$ and $\delta_{g} / h_{0}$, depend on three dimensionless parameters, $\lambda / h_{0}, \delta_{s} / h_{0}$, and $\Gamma_{0} h_{0}^{2} / D_{N}$. The first two parameters characterize the surface roughness, and the third represents the competition between the adhesive interaction and the bending stiffness of the membrane.

For quantitative analysis, we take representative values for the van der Waals interaction by setting $h_{0}=0.6 \mathrm{~nm}$ and $\Gamma_{0}=0.45 \mathrm{~J} / \mathrm{m}^{2}$. Atomic force microscopy (AFM) measurements have reported the values of $h_{0}$ ranging from 0.4 to $0.9 \mathrm{~nm}$ for graphene on $\mathrm{SiO}_{2}{ }^{2,12,13}$ The intrinsic adhesion energy is taken to be the value measured by Koenig et al. ${ }^{1}$ for the monolayer graphene. For the bending modulus, we note that by the classical theory the modulus scales with the cube of the membrane thickness, i.e., $D \sim t^{3}$. However, such a scaling does not apply for monolayer graphene. The physical mechanism that leads to a finite bending modulus for monolayer graphene is fundamentally different from that in the classical theory. ${ }^{14}$ An intrinsic bending modulus has been theoretically predicted for monolayer graphene, ${ }^{14-17}$ irrespective of its thickness. For a membrane containing an arbitrary number of graphene layers, the bending modulus is approximately given by ${ }^{16}$

$$
D_{N}=N D_{1}+E s^{3}\left(N^{3}-N\right) / 12
$$

where $N$ is the number of layers, $E$ is the in-plane Young's modulus of graphene, and $s$ is the spacing between adjacent graphene layers. The first term on the right-hand side of Eq. (6) accounts for the intrinsic bending stiffness of each graphene monolayer, whereas the second term is the contribution from the in-plane stiffness. Taking $D_{1}=1.61 \mathrm{eV},{ }^{16} E=1.0 \mathrm{TPa},{ }^{5}$ and $s=0.34$ $\mathrm{nm}$, Eq. (6) predicts that the bending modulus is $126 \mathrm{eV}$ for a bilayer graphene $(N=2)$ and 496 eV for a trilayer graphene $(N=3)$; Density-functional tight-binding calculations predicted that $D_{2}=180 \mathrm{eV}$ and $D_{3}=690 \mathrm{eV} .{ }^{16}$ Remarkably, from monolayer to bilayer, the bending modulus 
increases by two orders of magnitude, which can be attributed to lack of the contribution from the in-plane stiffness for bending of a monolayer. For $N>>1$, the effect of in-plane stiffness dominates and the bending modulus recovers the classical behavior with $D_{N} \approx E s^{3} N^{3} / 12=E t^{3} / 12$.

Figure 2 plots the normalized mean separation $\left(h_{m}\right)$ and corrugation amplitude $\left(\delta_{g}\right)$ for graphene membranes with $N=1,2,3$, and 10 , taking $\delta_{s}=0.2 \mathrm{~nm}$ based on the surface roughness measurements by Koenig et al. ${ }^{1}$ For each case, a transition from conformal to non-formal morphology occurs as the corrugation wavelength decreases. At the long wavelength limit the mean separation approaches $h_{0}$ and the corrugation amplitude of graphene approaches that of the surface. Thus, the graphene is conformal to the surface. For short wavelengths, however, the corrugation amplitude of graphene decreases and approaches zero to accommodate the bending energy. Meanwhile, the mean separation increases. A sharp transition occurs at a critical wavelength that depends on the bending modulus of the membrane. Similar transition has been predicted in previous studies. ${ }^{10,11}$

At the equilibrium morphology, the total free energy is negative, and the work of adhesion is calculated as $\Gamma=-\tilde{U}_{\text {total }}\left(h_{m}, \delta_{g}\right)$. As shown in Fig. 3, the transition of morphology leads to a similar transition in the work of adhesion. When the graphene membrane is conformal to the corrugated surface $\left(\delta_{g} / \delta_{s} \sim 1\right.$ ), the work of adhesion is close to the intrinsic adhesion energy $\left(\Gamma \sim \Gamma_{0}\right)$. On the other hand, when the graphene membrane is non-conformal ( $\delta_{g} / \delta_{s} \rightarrow 0$ ), the work of adhesion drops considerably. We note that the work of adhesion includes a small contribution from the elastic bending energy in addition to the van der Waals interaction energy. 
In general, the morphology and the work of adhesion depend on both the corrugation wavelength and amplitude. An arbitrarily rough surface may be considered as having many Fourier components, with different wavelengths and amplitudes. A graphene membrane on top would be conformal to the long-wavelength modes only, resulting in longer correlation length and smaller root-mean-square (RMS) amplitude in comparison to the bare surface. Experimentally measured correlation functions and RMS have indeed shown the same trend. ${ }^{1,2}$ However, a quantitative analysis for an arbitrarily rough surface would require a statistical approach, which is beyond the scope of the present study.

With a sinusoidal surface corrugation, Fig. 2 shows that the monolayer graphene remains nearly conformal for corrugation wavelengths greater than a few times the equilibrium separation (e.g., $\lambda / h_{0}>5$ ). For a bilayer graphene, it becomes nearly flat (thus non-conformal) at $\lambda / h_{0} \sim 12$. Thus, for a corrugation wavelength in between $\left(5<\lambda / h_{0}<12\right)$, the monolayer graphene is conformal while the bilayer and other graphene membranes $(N>1)$ are nonconformal. As a result, the work of adhesion for the monolayer graphene would be close to the intrinsic adhesion energy $\left(\Gamma \approx \Gamma_{0}\right)$, while all the multilayered graphene membranes would have considerably lower work of adhesion.

Figure 4 plots the work of adhesion as a function of $N$ for $\lambda / h_{0}=10$ and three different corrugation amplitudes. The work of adhesion decreases with increasing $N$, with a lower bound set by the limiting case when the membrane remains flat on the corrugated surface. The lower bound value for the work of adhesion decreases with increasing amplitude of surface corrugation. However, the present analysis is limited to relatively low amplitudes (e.g., $\delta_{s} / h_{0}<0.5$ ), since higher-order terms would have to be included in the free energy function for large amplitudes. Nevertheless, with $\delta_{s}=0.2 \mathrm{~nm}$, the work of adhesion drops from $0.45 \mathrm{~J} / \mathrm{m}^{2}$ for the monolayer 
graphene to $0.37 \mathrm{~J} / \mathrm{m}^{2}$ for the bilayer graphene, in reasonable agreement with the measurements by Koenig et al. ${ }^{1}$ Furthermore, the work of adhesion is nearly identical for all the multilayered graphene $(N>1)$, also in agreement with the experiment.

In summary, the effects of surface roughness on the morphology and adhesion energy of substrate-supported graphene membranes are analyzed by a theoretical model of van der Waals interaction. The results agree reasonably well with the experimental measurements for graphene on silicon oxide. The drastic difference in the bending moduli for the monolayer and multilayered graphene membranes leads to a transition in the morphology of the membranes on a corrugated surface, which in turn leads to a considerable difference in the measured adhesion energy. Moreover, the theoretical results suggest a possible approach to tunable adhesion of graphene membranes. While the work of adhesion has a fixed upper bound set by the intrinsic adhesion energy, the lower bound is tunable by controlling the amplitude of surface corrugation. Between the two bounds, the work of adhesion varies with the corrugation wavelength and the number of graphene layers.

\section{Acknowledgments}

The authors gratefully acknowledge funding of this work by National Science Foundation through Grant No. 0926851. 


\section{References}

1. S. P. Koenig, N. G. Boddeti, M. L. Dunn and J. S. Bunch, Nature Nanotech. (Published online, 14 August 2011), DOI: 10.1038/nnano.2011.123.

2. M. Ishigami, J. H. Chen, W. G. Cullen, M. S. Fuhrer and E. D. Willams, Nano Lett. 7, 1643 (2007).

3. N. T. Cuong, M. Otani and S. Okada, Phys. Rev. Lett. 106, 106801 (2011).

4. Z. Zong, C. Chen, M. R. Dokmeci and K. Wan, J. Appl. Phys. 107, 026104 (2010).

5. C. Lee, X. Wei, J.W. Kysar, J. Hone, Science 321, 385 (2008).

6. R. Al-Jishi, G. Dresselhaus, Phys. Rev. B 26, 4514-4522 (1982).

7. Y.-J. Kang, J. Kang, K.J. Chang, Phys. Rev. B 78, 115404 (2008).

8. M.Z. Hossain, Appl. Phys. Lett. 95, 143125 (2009).

9. Z. Lu, M.L. Dunn, J. Appl. Phys. 107, 044301 (2010).

10. T. Li and Z. Zhang, J. Phys. D: Appl. Phys. 43, 075303 (2010).

11. Z. H. Aitken and R. Huang, J. Appl. Phys. 107, 123531 (2010).

12. A. Gupta, G. Chen, P. Joshi, S. Tadigadapa, and P. C. Eklund, Nano Lett. 6, 2667 (2006).

13. S. Sonde, F. Giannazzo, V. Raineri and E. Rimini, J. Vac. Sci. Technol. B 27, 868 (2009).

14. Q. Lu, M. Arroyo and R. Huang, J. Phys. D: Appl. Phys. 42, 102002 (2009).

15. K. N. Kudin, G. E. Scuseria, and B. I. Yakobson, Phys. Rev. B 64235406 (2001).

16. P. Koskinen and O. O. Kit, Phys. Rev. B 82, 235420 (2010).

17. D. Sen, K.S. Novoselov, P.M. Reis, M.J. Buehler, Small 6, 1108 (2010). 


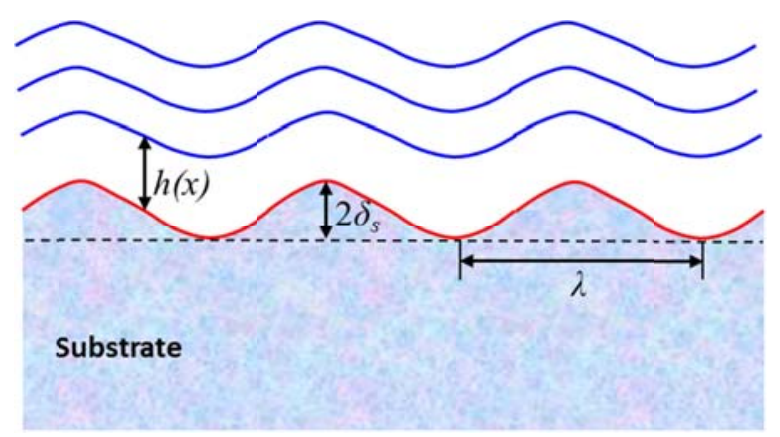

Figure 1. Schematic illustration of a trilayer graphene membrane on a corrugated substrate surface. 


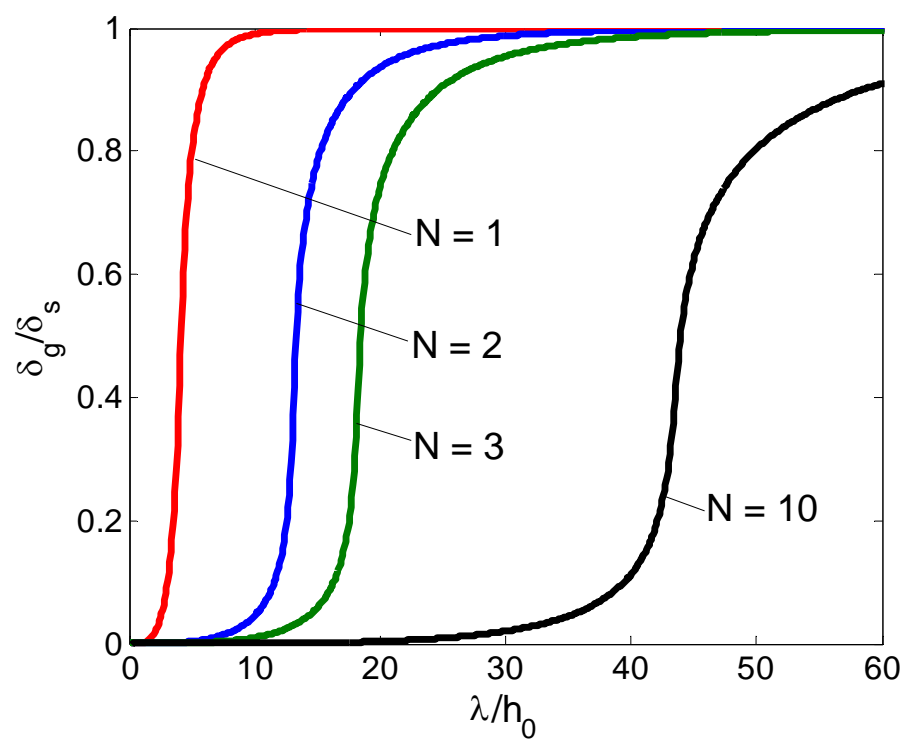

(a)

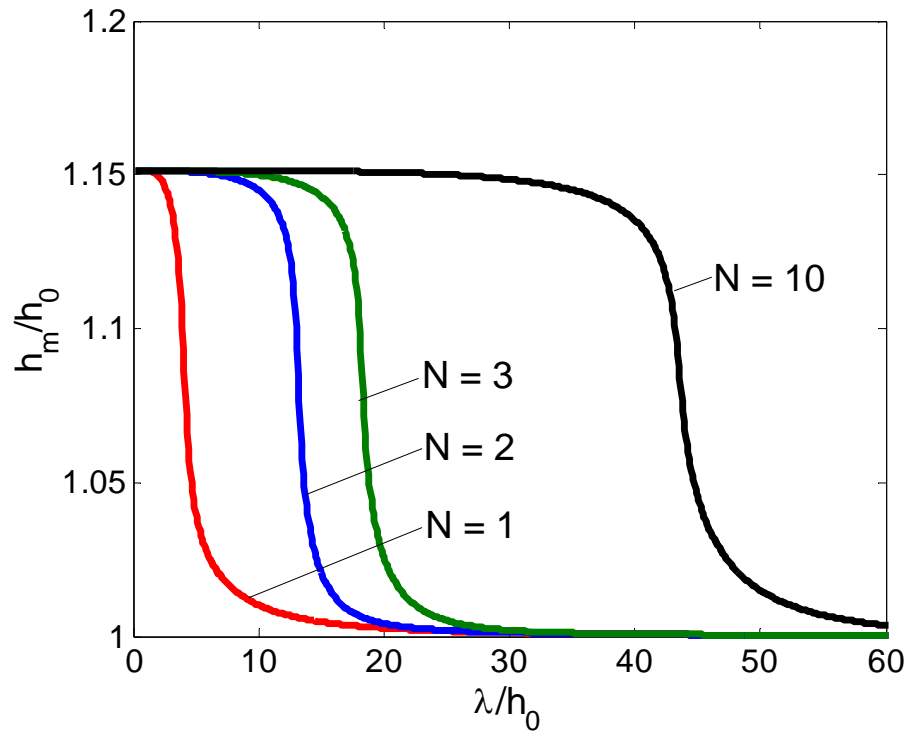

(b)

Figure 2. Equilibrium corrugation of graphene: (a) amplitude and (b) mean separation, as functions of the corrugation wavelength. 


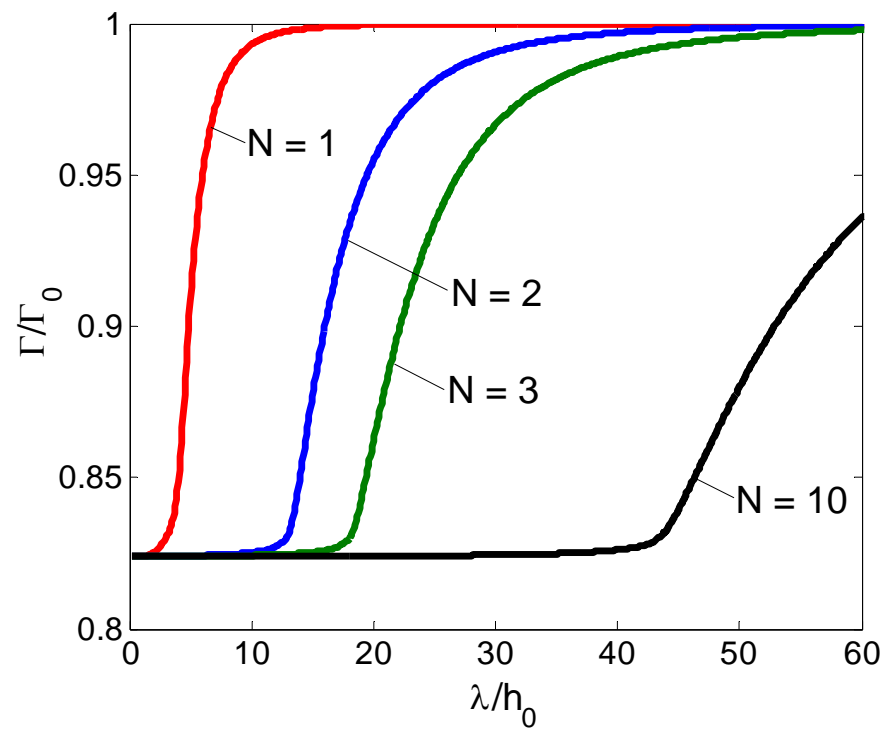

Figure 3. Normalized work of adhesion for graphene membranes on a corrugated surface. 


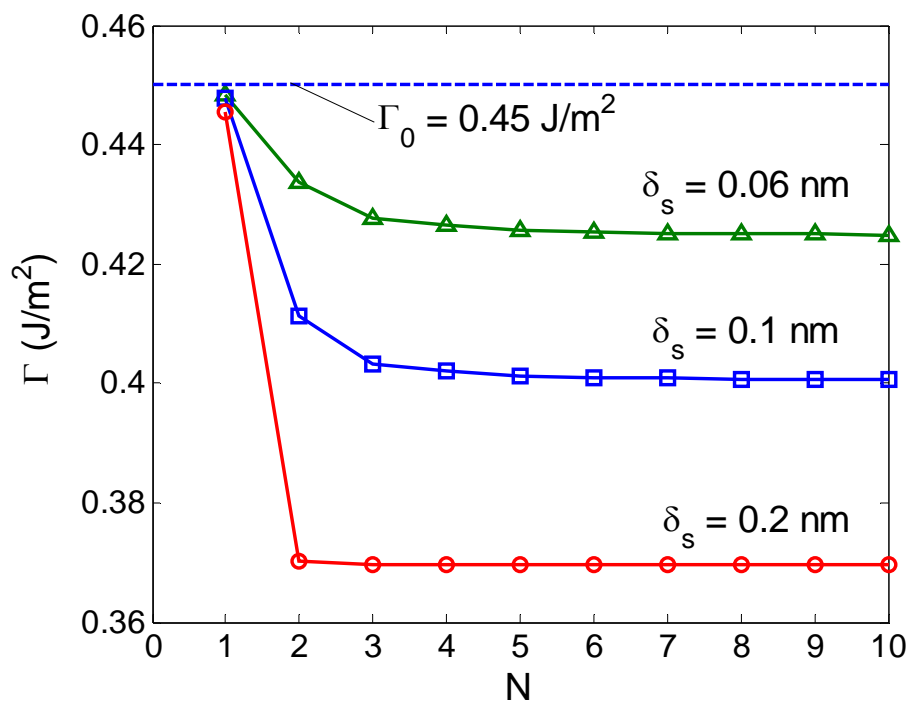

Figure 4. Work of adhesion for graphene membranes with 1 to 10 layers on corrugated surfaces of different corrugation amplitudes. 